\title{
GEOMETRIC AND HOMOTOPY THEORETIC METHODS IN NIELSEN COINCIDENCE THEORY
}

\author{
ULRICH KOSCHORKE
}

Received 30 November 2004; Accepted 21 July 2005

In classical fixed point and coincidence theory, the notion of Nielsen numbers has proved to be extremely fruitful. Here we extend it to pairs $\left(f_{1}, f_{2}\right)$ of maps between manifolds of arbitrary dimensions. This leads to estimates of the minimum numbers $\operatorname{MCC}\left(f_{1}, f_{2}\right)$ (and $\mathrm{MC}\left(f_{1}, f_{2}\right)$, resp.) of path components (and of points, resp.) in the coincidence sets of those pairs of maps which are $\left(f_{1}, f_{2}\right)$. Furthermore we deduce finiteness conditions for $\operatorname{MC}\left(f_{1}, f_{2}\right)$. As an application, we compute both minimum numbers explicitly in four concrete geometric sample situations. The Nielsen decomposition of a coincidence set is induced by the decomposition of a certain path space $E\left(f_{1}, f_{2}\right)$ into path components. Its higher-dimensional topology captures further crucial geometric coincidence data. An analoguous approach can be used to define also Nielsen numbers of certain link maps.

Copyright (C 2006 Ulrich Koschorke. This is an open access article distributed under the Creative Commons Attribution License, which permits unrestricted use, distribution, and reproduction in any medium, provided the original work is properly cited.

\section{Introduction and discussion of results}

Throughout this paper $f_{1}, f_{2}: M \rightarrow N$ denote two (continuous) maps between the smooth connected manifolds $M$ and $N$ without boundary, of strictly positive dimensions $m$ and $n$, respectively, $M$ being compact.

We would like to measure how small (or simple in some sense) the coincidence locus

$$
C\left(f_{1}, f_{2}\right):=\left\{x \in M \mid f_{1}(x)=f_{2}(x)\right\}
$$

can be made by deforming $f_{1}$ and $f_{2}$ via homotopies. Classically one considers the minimum number of coincidence points

$$
\operatorname{MC}\left(f_{1}, f_{2}\right):=\min \left\{\# C\left(f_{1}^{\prime}, f_{2}^{\prime}\right) \mid f_{1}^{\prime} \sim f_{1}, f_{2}^{\prime} \sim f_{2}\right\}
$$

(cf. [1], (1.1)). It coincides with the minimum number $\min \left\{\# C\left(f_{1}^{\prime}, f_{2}\right) \mid f_{1}^{\prime} \sim f_{1}\right\}$ where only $f_{1}$ is modified by a homotopy (cf. [2]). In particular, in topological fixed point theory 
(where $M=N$ and $f_{2}$ is the identity map) this minimum number is the principal object of study (cf. [3, page 9]).

In higher codimensions, however, the coincidence locus is generically a manifold of dimension $m-n>0$, and $\mathrm{MC}\left(f_{1}, f_{2}\right)$ is often infinite (see, e.g., Examples 1.4 and 1.6 below). Thus it seems more meaningful to study the minimum number of coincidence components

$$
\operatorname{MCC}\left(f_{1}, f_{2}\right):=\min \left\{\# \pi_{0}\left(C\left(f_{1}^{\prime}, f_{2}^{\prime}\right)\right) \mid f_{1}^{\prime} \sim f_{1}, f_{2}^{\prime} \sim f_{2}\right\}
$$

where $\# \pi_{0}\left(C\left(f_{1}^{\prime}, f_{2}^{\prime}\right)\right)$ denotes the (generically finite) number of path components of the indicated coincidence subspace of $M$.

Question 1.1. How big are $\operatorname{MCC}\left(f_{1}, f_{2}\right)$ and $\operatorname{MC}\left(f_{1}, f_{2}\right)$ ? In particular, when do these invariants vanish, that is, when can the maps $f_{1}$ and $f_{2}$ be deformed away from one another?

In this paper, we discuss lower bounds for $\operatorname{MCC}\left(f_{1}, f_{2}\right)$ and geometric obstructions to $\operatorname{MC}\left(f_{1}, f_{2}\right)$ being trivial or finite.

A careful investigation of the differential topology of generic coincidence submanifolds yields the normal bordism classes (cf. (4.6) and (4.7))

$$
\begin{gathered}
\omega\left(f_{1}, f_{2}\right) \in \Omega_{m-n}(M ; \varphi), \\
\tilde{\omega}\left(f_{1}, f_{2}\right) \in \Omega_{m-n}\left(E\left(f_{1}, f_{2}\right) ; \tilde{\varphi}\right)
\end{gathered}
$$

as well as a sharper ("nonstabilized") version

$$
\omega^{\#}\left(f_{1}, f_{2}\right) \in \Omega^{\#}\left(f_{1}, f_{2}\right)
$$

of $\widetilde{\omega}\left(f_{1}, f_{2}\right)$ (cf. Remark 4.2). Here the path space

$$
E\left(f_{1}, f_{2}\right):=\left\{(x, \theta) \in M \times N^{I} \mid \theta(0)=f_{1}(x), \theta(1)=f_{2}(x)\right\}
$$

(cf. Section 2), also known as (a kind of) homotopy equalizer of $f_{1}$ and $f_{2}$, plays a crucial role. In general it has a very rich topology involving both $M$ and the loop space of $N$. Already the set $\pi_{0}\left(E\left(f_{1}, f_{2}\right)\right)$ of path components can be huge-it corresponds bijectively to the Reidemeister set

$$
R\left(f_{1}, f_{2}\right)=\pi_{1}(N) / \text { Reidemeister equivalence }
$$

(cf. [1, 3.1] and our Proposition 2.1 below) which is of central importance in classical Nielsen theory. Thus it is only natural to define a "Nielsen number" $N\left(f_{1}, f_{2}\right)$ (and a sharper version $N^{\#}\left(f_{1}, f_{2}\right)$, resp.) to be the number of those ("essential") path components which contribute nontrivially to the bordism class $\widetilde{\omega}\left(f_{1}, f_{2}\right)$ (and to $\omega^{\#}\left(f_{1}, f_{2}\right)$, resp.), compare Definition 4.1 and Remark 4.2. 
Theorem 1.2. (i) The integers $N\left(f_{1}, f_{2}\right)$ and $N^{\#}\left(f_{1}, f_{2}\right)$ depend only on the homotopy classes of $f_{1}$ and $f_{2}$; (ii) $N\left(f_{1}, f_{2}\right)=N\left(f_{2}, f_{1}\right)$ and $N^{\#}\left(f_{1}, f_{2}\right)=N^{\#}\left(f_{2}, f_{1}\right)$; (iii) $0 \leq N\left(f_{1}\right.$, $\left.f_{2}\right) \leq N^{\#}\left(f_{1}, f_{2}\right) \leq \operatorname{MCC}\left(f_{1}, f_{2}\right) \leq \operatorname{MC}\left(f_{1}, f_{2}\right)$; if $n \neq 2$, then also $\operatorname{MCC}\left(f_{1}, f_{2}\right) \leq \# R\left(f_{1}, f_{2}\right)$; (iv) if $m=n$, then $N\left(f_{1}, f_{2}\right)=N^{\#}\left(f_{1}, f_{2}\right)$ coincides with the classical Nielsen number (cf. [1, Definition 3.6]).

Remark 1.3. In various situations, some of the estimates spelled out in part (iii) of this theorem are known to be sharp (compare also [12]). For example, in the self-coincidence setting (where $f_{1}=f_{2}$ ) we have always $\operatorname{MCC}\left(f_{1}, f_{2}\right) \leq 1$ (since here $C\left(f_{1}, f_{2}\right)=M$ ). In the "root setting" (where $f_{2}$ maps to a constant value $* \in N$ ) all Nielsen classes are simultaneously essential or inessential (since our $\omega$-invariants are always compatible with homotopies of $\left(f_{1}, f_{2}\right)$ and hence, in this particular case, with the action of $\pi_{1}(N, *)$, cf. the discussion in [12] following (1.10)). Therefore in both settings $\operatorname{MCC}\left(f_{1}, f_{2}\right)$ is equal to the Nielsen number $N\left(f_{1}, f_{2}\right)$ provided $\widetilde{\omega}\left(f_{1}, f_{2}\right) \neq 0$ (and $n \neq 2$ if $f_{2} \equiv *$ ).

Further geometric and homotopy theoretic considerations allow us to determine the Nielsen and minimum numbers explicitly in several concrete sample situations (for proofs see Section 6 below).

Example 1.4. Given integers $q>1$ and $r$, let $N=\mathbb{C} P(q)$ be $q$-dimensional complex projective space, let $M=S\left(\otimes_{\mathbb{C}}^{r} \lambda_{\mathbb{C}}\right)$ be the total space of the unit circle bundle of the $r$ th tensor power of the canonical complex line bundle, and let $f: M \rightarrow N$ denote the fiber projection. Then

$$
\begin{gathered}
N(f, f)=N^{\#}(f, f)=\operatorname{MCC}(f, f)= \begin{cases}0 & \text { if } q \equiv-1(r), q \equiv 1(2), \\
1 & \text { else; }\end{cases} \\
\operatorname{MC}(f, f)= \begin{cases}0 & \text { if } q \equiv-1(r), q \equiv 1(2), \\
1 & \text { if } q \equiv-1(r), q \equiv 0(2), \\
\infty & \text { if } q \equiv-1(r) .\end{cases}
\end{gathered}
$$

As was shown above (cf. Remark 1.3), in any self-coincidence situation (where $f_{1}=f_{2}$ ) $\operatorname{MCC}\left(f_{1}, f_{2}\right)$ must be 0 or 1 and it remains only to decide which value occurs. In the previous example this can be settled by the normal bordism class $\omega(f, f) \in \Omega_{1}(M ; \varphi)$, a weak form of $\tilde{\omega}(f, f)$ which, however, captures a delicate ("second order") $\mathbb{Z}_{2}$-aspect as well as the dual of the classical first order obstruction. Already in this simple case standard methods of singular (co)homology theory yield only a necessary condition for $\operatorname{MCC}\left(f_{1}, f_{2}\right)$ to vanish (cf. $\left.[7,2.2]\right)$. In higher codimensions $m-n$ the advantage of the normal bordism approach can be truely dramatic.

Example 1.5. Given natural numbers $k<r$, let $M=V_{r, k}$ (and $N=G_{r, k}$, resp.) be the Stiefel manifold of orthonormal $k$-frames (and the Grassmannian of $k$-planes, resp.) in $\mathbb{R}^{r}$. Let $f: M \rightarrow N$ map a frame to the plane it spans.

Assume $r \geq 2 k \geq 2$. Then

$$
N(f, f)=N^{\#}(f, f)=\operatorname{MCC}(f, f)=\operatorname{MC}(f, f)= \begin{cases}0 & \text { if } \omega(f, f)=0, \\ 1 & \text { else. }\end{cases}
$$


Here the normal bordism obstruction $\omega(f, f) \in \Omega_{m-n}(M ; \varphi)$ (cf. (4.7)) contains precisely as much information as its "highest order component"

$$
2 \chi\left(G_{r, k}\right) \cdot[\mathrm{SO}(k)] \in \Omega_{m-n}^{\mathrm{fr}} \cong \pi_{m-n}^{S}
$$

where $[\mathrm{SO}(k)]$ denotes the framed bordism class of the Lie group $\mathrm{SO}(k)$, equipped with a left invariant parallelization; the Euler number $\chi\left(G_{r, k}\right)$ is easily calculated: it vanishes if $k \not \equiv r \equiv 0(2)$ and equals $\left(\begin{array}{c}{[r / 2]} \\ {[k / 2]}\end{array}\right)$ otherwise. Without loosing its geometric flavor, our original question translates here-via the Pontryagin-Thom isomorphism-into deep problems of homotopy theory (compare the discussion in the introduction of [11]). Fortunately powerful methods are available in homotopy theory which imply, for example, that $\operatorname{MCC}(f, f)=\operatorname{MC}(f, f)=0$ if $k$ is even or $k=7$ or 9 or $\chi\left(G_{r, k}\right) \equiv 0(12)$; however, if $k=1$ and $r \equiv 1(2)$, or if $k=3$ and $r \not \equiv 1(12)$ is odd, or if $k=5$ and $r \equiv 5(6)$, then $\operatorname{MCC}(f, f)=\operatorname{MC}(f, f)=1$.

These results seem to be entirely out of the reach of the methods of singular (co)homology theory since we would have to deal here with obstructions of order $m-$ $n+1=k(k-1) / 2+1$.

Example 1.6. Let $N$ be the torus $\left(S^{1}\right)^{n}$ and let $\iota_{1}, \ldots, \iota_{n}$ denote the canonical generators of $H^{1}\left(\left(S^{1}\right)^{n} ; \mathbb{Z}\right)$. If the homomorphism

$$
f_{1 *}-f_{2 *}: H_{1}(M ; \mathbb{Z}) \longrightarrow H_{1}\left(\left(S^{1}\right)^{n} ; \mathbb{Z}\right)
$$

has an infinite cokernel (or, equivalently, the rank of its image is strictly smaller than $n$ ), then

$$
N\left(f_{1}, f_{2}\right)=N^{\#}\left(f_{1}, f_{2}\right)=\operatorname{MCC}\left(f_{1}, f_{2}\right)=\operatorname{MC}\left(f_{1}, f_{2}\right)=0 .
$$

On the other hand, if the cup product

$$
\prod_{j=1}^{n}\left(f_{1}^{*}-f_{2}^{*}\right)\left(\iota_{j}\right) \in H^{n}(M ; \mathbb{Z})
$$

is nontrivial, then $\operatorname{MC}\left(f_{1}, f_{2}\right)=\infty$ whenever $m>n$; if in addition $n \neq 2$, then $\operatorname{MCC}\left(f_{1}, f_{2}\right)$ equals the (finite) cardinality of the cokernel of $f_{1 *}-f_{2 *}$ (cf.(1.11)).

In the special case when $N$ is the unit circle $S^{1}$ we have: $\operatorname{MCC}\left(f_{1}, f_{2}\right)=\operatorname{MC}\left(f_{1}, f_{2}\right)=$ 0 if $f_{1}$ is homotopic to $f_{2}$; otherwise $\operatorname{MCC}\left(f_{1}, f_{2}\right)=\#$ coker $\left(f_{1 *}-f_{2 *}\right)$, but (if $m>1$ ) $\operatorname{MC}\left(f_{1}, f_{2}\right)=\infty$. 
An important special case of our invariants are the degrees

$$
\operatorname{deg}^{\sharp}(f):=\omega^{\#}(f, *), \quad \widetilde{\operatorname{deg}}(f):=\widetilde{\omega}(f, *), \quad \operatorname{deg}(f):=\omega(f, *)
$$

of a given map $f: M \rightarrow N$ (here $*$ denotes a constant map).

Example 1.7 (homotopy groups). Let $M$ be the sphere $S^{m}$; in view of the previous example we may also assume that $n \geq 2$.

Then, given $\left[f_{i}\right] \in \pi_{m}\left(N, *_{i}\right), i=1,2, *_{1} \neq *_{2}$, we can identify $\Omega^{\#}\left(f_{1}, f_{2}\right), \Omega_{m-n}\left(E\left(f_{1}\right.\right.$, $\left.\left.f_{2}\right) ; \tilde{\varphi}\right)$ and $\Omega_{m-n}(M ; \varphi)$ with the corresponding groups in the top line of the diagram

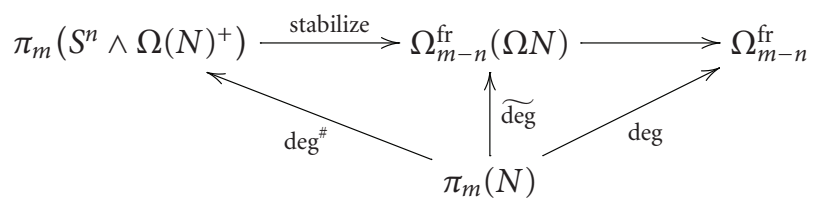

(This is possible since the loop space $\Omega N$ occurs as a typical fiber of the natural projection $p: E\left(f_{1}, f_{2}\right) \rightarrow S^{m}$, cf. [12, Section 7], and [13].)

Furthermore, after deforming the maps $f_{1}$ and $f_{2}$ until they are constant on opposite half spheres in $S^{n}$, we see that

$$
\widetilde{\omega}\left(f_{1}, f_{2}\right)=\widetilde{\omega}\left(f_{1}, *_{2}\right)+\widetilde{\omega}\left(*_{1}, f_{2}\right),
$$

and similarly for $\omega^{\#}$ and $\omega$.

Thus it suffices to study the degree maps in diagram (1.15). They turn out to be group homomorphisms which commute with the indicated natural forgetful homomorphisms.

It can be shown (cf. [13]) that $\operatorname{deg}^{\#}(f)$ is (a strong version of) the Hopf-Ganea invariant of $[f] \in \pi_{m}(N)$ (w.r. to the attaching map of a top cell in $N$, compare $[5,6.7]$ ), while $\widetilde{\operatorname{deg}}(f)$ is closely related to (weaker) stabilized Hopf-James invariants ([12, 1.14]).

Special case: $M=S^{m}, N=S^{n}, n \geq 2$. Here deg ${ }^{\#}$ is injective and we see that

$$
N(f, *) \leq N^{\#}(f, *)=\operatorname{MCC}(f, *)= \begin{cases}0 & \text { if } f \text { is null homotopic, } \\ 1 & \text { otherwise }\end{cases}
$$

for all maps $f: S^{m} \rightarrow S^{n}$. There are many dimension combinations $(m, n)$, where the equality $N(f, *)=N^{\#}(f, *)$ is also valid for all $f$ or, equivalently, where deg is injective (compare, e.g., our Remark 4.2 below or $[12,1.16])$. However, if $n \neq 1,3,7$ is odd and $m=2 n-1$, or if, for example, $(m, n)=(8,4),(9,4),(9,3),(10,4),(16,8),(17,8),(10+n, n)$ for $3 \leq n \leq 11$, or $(24,6)$, then there exists a map $f: S^{m} \rightarrow S^{n}$ such that $0=N(f, *)<$ $N^{\#}(f, *)=1$ (compare $\left.[12,1.17]\right)$.

Very special case: $M=S^{3}, N=S^{2}$. Here

$$
\widetilde{\operatorname{deg}}: \pi_{3}\left(S^{2}\right) \cong \mathbb{Z} \longrightarrow \Omega_{1}^{\text {fr }}\left(\Omega S^{2}\right) \cong \mathbb{Z}_{2} \oplus \mathbb{Z}
$$


captures the Freudenthal suspension and the classical Hopf invariant of a homotopy class $[f]$; therefore $\widetilde{\mathrm{deg}}$ is injective (and so is $\mathrm{deg}^{\#}$ a fortiori).

On the other hand the invariant $\operatorname{deg}(f) \in \Omega_{1}^{\mathrm{fr}} \cong \mathbb{Z}_{2}$ (which does not involve the path space $E(f, *))$ retains only the suspension of $f$. The corresponding homological invariant $\mu(\operatorname{deg}(f)) \in H_{1}\left(S^{3} ; \mathbb{Z}\right)$ vanishes altogether.

Finally let us point out that our approach can also be applied fruitfully to study linking phenomena. Consider, for example, a link map

$$
f=f_{1} \amalg f_{2}: M_{1} \amalg M_{2} \longrightarrow N \times \mathbb{R}
$$

(i.e., the closed manifolds $M_{1}$ and $M_{2}$ have disjoint images). Just as in the case of two disjoint closed curves in $\mathbb{R}^{3}$ the degree of linking can be measured to some extend by the geometry of the overcrossing locus: it consists of that part of the coincidence locus of the projections to $N$, where $f_{1}$ is bigger than $f_{2}$ (w.r. to the $\mathbb{R}$-coordinate). Here the normal bordism/path space approach yields strong unlinking obstructions which, in addition, turn out to distinguish a great number of different link homotopy classes. Moreover it leads to a natural notion of Nielsen numbers for link maps (cf. [10]).

\section{The path space $E\left(f_{1}, f_{2}\right)$}

A crucial feature of our approach to Nielsen theory is the central role played by the space $E\left(f_{1}, f_{2}\right)$. It yields the Nielsen decomposition of coincidence sets in a very natural geometric fashion. In the defining (1.6) $N^{I}$ denotes the space of all continuous paths $\theta: I:=[0,1] \rightarrow N$ with the compact-open topology. The starting point/endpoint fibration $N^{I} \rightarrow N \times N$ pulls back, via the map

$$
\left(f_{1}, f_{2}\right): M \longrightarrow N \times N
$$

to yield the Hurewicz fibration

$$
p: E\left(f_{1}, f_{2}\right) \longrightarrow M
$$

defined by $p(x, \theta)=x$. Given a coincidence point $x_{0} \in M$, the fiber $p^{-1}\left(\left\{x_{0}\right\}\right)$ is just the loop space $\Omega\left(N, y_{0}\right)$ of paths in $N$ starting and ending at $y_{0}=f_{1}\left(x_{0}\right)=f_{2}\left(x_{0}\right)$; let $\theta_{0}$ denote the constant path at $y_{0}$.

Proposition 2.1. The sequence of group homomorphisms

$$
\begin{aligned}
\cdots & \longrightarrow \pi_{k+1}\left(M, x_{0}\right) \stackrel{f_{1 *}-f_{2 *}}{\longrightarrow} \pi_{k+1}\left(N, y_{0}\right) \stackrel{\text { incl }_{*}}{\longrightarrow} \pi_{k}\left(E\left(f_{1}, f_{2}\right),\left(x_{0}, \theta_{0}\right)\right) \\
& \stackrel{p_{*}}{\longrightarrow} \pi_{k}\left(M, x_{0}\right) \longrightarrow \cdots \longrightarrow \pi_{1}\left(M, x_{0}\right)
\end{aligned}
$$

is exact. Moreover, the fiber inclusion incl : $\Omega\left(N, y_{0}\right) \rightarrow E\left(f_{1}, f_{2}\right)$ induces a bijection of the sets

$$
R\left(f_{1}, f_{2}\right)=\pi_{1}\left(N, y_{0}\right) / \text { Reidemeister equivalence } \longrightarrow \pi_{0}\left(E\left(f_{1}, f_{2}\right)\right),
$$

where two classes $[\theta],\left[\theta^{\prime}\right] \in \pi_{1}\left(N, y_{0}\right)=\pi_{0}\left(\Omega\left(N, y_{0}\right)\right)$ are called Reidemeister equivalent if $\left[\theta^{\prime}\right]=f_{1 *}(\tau)^{-1} \cdot[\theta] \cdot f_{2 *}(\tau)$ for some $\tau \in \pi_{1}\left(M, x_{0}\right)$. 
The proof is fairly evident. In fact, we are dealing here essentially with the long exact homotopy sequence of the fibration $p$.

\section{Normal bordism}

In this section we recall some standard facts about a geometric language which seems well suited to describe relevant coincidence phenomena in arbitrary codimensions.

Let $X$ be a topological space and let $\varphi$ be a virtual real vector bundle over $X$, that is, an ordered pair $\left(\varphi^{+}, \varphi^{-}\right)$of vector bundles written $\varphi=\varphi^{+}-\varphi^{-}$.

A singular $\varphi$-manifold in $X$ of dimension $q$ is a triple $(C, g, \bar{g})$, where

(i) $C$ is a closed smooth $q$-dimensional manifold;

(ii) $g: C \rightarrow X$ is a continuous map;

(iii) $\bar{g}$ : TC $\oplus g^{*}\left(\varphi^{+}\right) \rightarrow g^{*}\left(\varphi^{-}\right)$is a stable vector bundle isomorphism (i.e., we can first add trivial vector bundles of suitable dimensions on both sides).

Two such triples $\left(C_{i}, g_{i}, \bar{g}_{i}\right), i=0,1$, are bordant if there exists a compact $\operatorname{singular}(q+$ 1)-dimensional $\varphi$-manifold $(B, b, \bar{b})$ in $X$ with boundary $\partial B=C_{0} \amalg C_{1}$ such that $b$ and $\bar{b}$, when restricted to $\partial B$, coincide with the corresponding data $g_{i}$ and $\bar{g}_{i}$ at $C_{i}, i=0,1$ (via vector fields pointing into $B$ along $C_{0}$ and out of $B$ along $C_{1}$ ). The resulting set of bordism classes, with the sum operation given by disjoint unions, is the qth normal bordism group $\Omega_{q}(X ; \varphi)$ of $X$ with coefficients in $\varphi$.

Example 3.1. Let $G$ denote the trivial group or the (special) orthogonal group $(S) O\left(q^{\prime}\right)$, $q^{\prime}>q+1$. For any topological space $Y$ let $\varphi^{+}$be the classifying bundle over $B G$, pulled back to $X=Y \times B G$, while $\varphi^{-}$is trivial. Then $\Omega_{q}(X ; \varphi)$ is the standard (stably) framed, oriented or unoriented $q$ th bordism group of $Y$ (cf., e.g., [4, I.4 and 8]).

For every virtual vector bundle $\varphi$ over a topological space $X$ there are well known Hurewicz homomorphisms

$$
\mu: \Omega_{q}(X ; \varphi) \rightarrow H_{q}\left(X ; \tilde{\mathbb{Z}}_{\varphi}\right), \quad q \in \mathbb{Z},
$$

into singular homology with local integer coefficients $\tilde{\mathbb{Z}}_{\varphi}$ (which are twisted like the orientation line bundle $\xi_{\varphi}=\xi_{\varphi^{+}} \otimes \xi_{\varphi^{-}}$of $\varphi$ ); they map a normal bordism class $[C, g, \bar{g}]$ to the image of the fundamental class $[C] \in H_{q}\left(C ; \widetilde{\mathbb{Z}}_{T C}\right)$ by the induced homomorphism $g_{*}$.

In most cases $\mu$ leads to a big loss of information. However for $q \leq 4$ this loss can often be measured so that explicit calculations of (and in) $\Omega_{q}(X ; \varphi)$ are possible (in particular so when $\varphi$ is highly nontrivial), see [9, Theorem 9.3]. We obtain for example, the following lemma.

Lemma 3.2. Assume $X$ is path connected. Then the following hold.

(i)

$$
\Omega_{0}(X ; \varphi) \underset{\stackrel{\mu}{\cong}}{\stackrel{\mu}{*}} H_{0}\left(X ; \tilde{\mathbb{Z}}_{\varphi}\right)= \begin{cases}\mathbb{Z} & \text { if } w_{1}(\varphi)=0, \\ \mathbb{Z}_{2} & \text { else. }\end{cases}
$$


(ii) The following sequence is exact:

$$
\Omega_{2}(X ; \varphi) \stackrel{\mu}{\longrightarrow} H_{2}\left(X ; \widetilde{\mathbb{Z}}_{\varphi}\right) \stackrel{w_{2}(\varphi)}{\longrightarrow} \mathbb{Z}_{2} \stackrel{\delta_{1}}{\longrightarrow} \Omega_{1}(X ; \varphi) \stackrel{\mu}{\longrightarrow} H_{1}\left(X ; \widetilde{\mathbb{Z}}_{\varphi}\right) \longrightarrow 0 .
$$

Here $\delta_{1}(1)$ is represented by the invariantly parallelized unit circle, together with a constant map, and

$$
\begin{aligned}
& w_{1}(\varphi)=w_{1}\left(\varphi^{+}\right)+w_{1}\left(\varphi^{-}\right) \\
& w_{2}(\varphi)=w_{2}\left(\varphi^{+}\right)+w_{1}\left(\varphi^{+}\right) w_{1}\left(\varphi^{-}\right)+w_{2}\left(\varphi^{-}\right)+w_{1}\left(\varphi^{-}\right)^{2}
\end{aligned}
$$

denote Stiefel-Whitney classes of $\varphi$.

The setting of (normal) bordism groups provides also a first rate illustration of the fact that the geometric and differential topology of manifolds on one hand, and homotopy theory on the other hand, are often but two sides of the same coin. Indeed, if $\varphi^{-}$ allows a complementary vector bundle $\varphi^{-\perp}$ (such that $\varphi^{-} \oplus \varphi^{-\perp}$ is trivial), then the wellknown Pontryagin-Thom construction allows us to interpret $\Omega_{q}(X ; \varphi), q \in \mathbb{Z}$, as a (stable) homotopy group of the Thom space of $\varphi^{+} \oplus \varphi^{-\perp}$ which consists of the total space of $\varphi^{+} \oplus \varphi^{-\perp}$ with one point "added at infinity" (compare, e.g., [4, I, 11 and 12]). Thus the methods of algebraic topology offer another (and often very powerful) approach to computing normal bordism groups (cf., e.g., [4, Chapter II]).

Example 3.3. The Thom space of the vector bundle $\varphi=\mathbb{R}^{k}$ over a one-point space is the sphere $S^{k}=\mathbb{R}^{k} \cup\{\infty\}$. Hence the framed bordism group $\Omega_{q}^{\mathrm{fr}}:=\Omega_{q}(\{$ point $\} ; \varphi)$ is canonically isomorphic to the stable homotopy group $\pi_{q}^{S}:=\lim _{k \rightarrow \infty} \pi_{q+k}\left(S^{k}\right)$ of spheres. It is computed and listed, for example, in Toda's tables (in [14, Chapter XIV]) whenever $q \leq 19$.

For further details and references concerning normal bordism see, for example, [6] or [9].

\section{The invariants}

In this section we discuss the invariants $\tilde{\omega}\left(f_{1}, f_{2}\right)$ and $N\left(f_{1}, f_{2}\right)$ based on normal bordism, as well as their sharper (nonstabilized) versions $\omega^{\#}\left(f_{1}, f_{2}\right)$ and $N^{\#}\left(f_{1}, f_{2}\right)$. We refer to [12] for some of the details and proofs (see also [13]).

In the special case when the map $\left(f_{1}, f_{2}\right): M \rightarrow N \times N$ is smooth and transverse to the diagonal

$$
\Delta=\{(y, y) \in N \times N \mid y \in N\}
$$

the coincidence set

$$
C=C\left(f_{1}, f_{2}\right)=\left(f_{1}, f_{2}\right)^{-1}(\Delta)=\left\{x \in M \mid f_{1}(x)=f_{2}(x)\right\}
$$




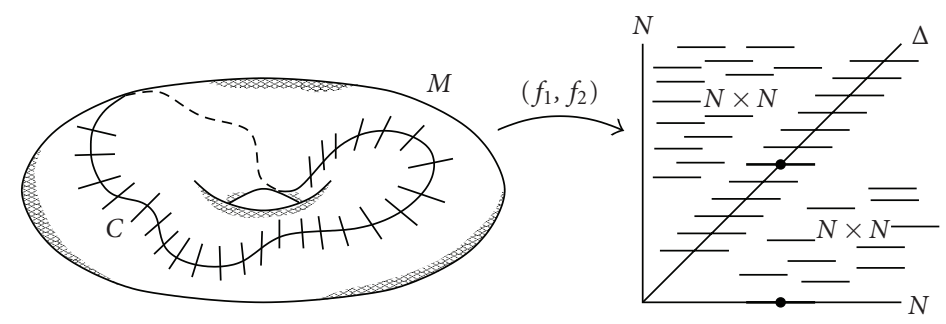

Figure 4.1. A generic coincidence manifold and its normal bundle.

is a smooth submanifold of $M$. It comes with the maps

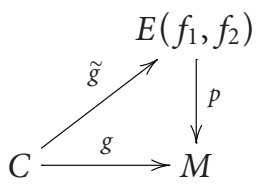

defined by $g(x)=x$ and $\tilde{g}(x)=\left(x\right.$, constant path at $\left.f_{1}(x)=f_{2}(x)\right), x \in C$.

The normal bundle of $C$ in $M$ is described by the isomorphism

$$
\nu(C, M) \cong\left(f_{1}, f_{2}\right)^{*}(\nu(\Delta, N \times N)) \cong f_{1}^{*}(T N) \mid C
$$

(see Figure 4.1) which yields

$$
\bar{g}: T C \oplus f_{1}^{*}(T N)|C \stackrel{\cong}{\longrightarrow} T M| C .
$$

Define

$$
\begin{gathered}
\tilde{\omega}\left(f_{1}, f_{2}\right):=[C, \tilde{g}, \bar{g}] \in \Omega_{m-n}\left(E\left(f_{1}, f_{2}\right) ; \tilde{\varphi}\right), \\
\omega\left(f_{1}, f_{2}\right):=[C, g, \bar{g}]=p_{*}\left(\widetilde{\omega}\left(f_{1}, f_{2}\right)\right) \in \Omega_{m-n}(M ; \varphi),
\end{gathered}
$$

where

$$
\varphi:=f_{1}^{*}(T N)-T M, \quad \tilde{\varphi}:=p^{*}(\varphi)
$$

Invariants with precisely the same properties can be constructed in general. Indeed, apply the preceding procedure to a smooth map $\left(f_{1}^{\prime}, f_{2}^{\prime}\right)$ which is transverse to $\Delta$ and approximates $\left(f_{1}, f_{2}\right)$.

Also apply the isomorphism $\Omega_{*}\left(E\left(f_{1}^{\prime}, f_{2}^{\prime}\right) ; \tilde{\varphi}^{\prime}\right) \cong \Omega_{*}\left(E\left(f_{1}, f_{2}\right) ; \tilde{\varphi}\right)$ induced by a small homotopy (cf. $[12,3.3])$ to $\widetilde{\omega}\left(f_{1}^{\prime}, f_{2}^{\prime}\right)$ in order to obtain $\widetilde{\omega}\left(f_{1}, f_{2}\right)$ and similarly $\omega\left(f_{1}, f_{2}\right)$. 
Now consider the decomposition

$$
\widetilde{\omega}\left(f_{1}, f_{2}\right)=\left\{\widetilde{\omega}_{A}\left(f_{1}, f_{2}\right)\right\} \in \Omega_{m-n}\left(E\left(f_{1}, f_{2}\right) ; \tilde{\varphi}\right)=\bigoplus_{A} \Omega_{m-n}(A ; \tilde{\varphi} \mid A)
$$

according to the various path components $A \in \pi_{0}\left(E\left(f_{1}, f_{2}\right)\right)$ of $E\left(f_{1}, f_{2}\right)$.

Definition 4.1. A pathcomponent of $E\left(f_{1}, f_{2}\right)$ is called essential if the corresponding direct summand of $\tilde{\omega}\left(f_{1}, f_{2}\right)$ is nontrivial. The Nielsen coincidence number $N\left(f_{1}, f_{2}\right)$ is the number of essential path components $A \in \pi_{0}\left(E\left(f_{1}, f_{2}\right)\right)$.

Since we assume $M$ to be compact, $N\left(f_{1}, f_{2}\right)$ is a finite integer. It vanishes if and only if $\tilde{\omega}\left(f_{1}, f_{2}\right)$ does.

Remark 4.2. In Figure 4.1 we have neglected an important geometric aspect: $C$ is much more than just an (abstract) singular manifold with an description of its stable normal bundle. If we keep track (i) of the fact that $C$ is a smooth submanifold in $M$, and (ii) of the nonstabilized isomorphism (4.4), we obtain the sharper invariants $\omega^{\#}\left(f_{1}, f_{2}\right)$ and $N^{\#}\left(f_{1}, f_{2}\right)$. Note, however, that the bordism set $\Omega^{\#}\left(f_{1}, f_{2}\right)$ in which $\omega^{\#}\left(f_{1}, f_{2}\right)$ lies has possibly no group structure - the union of submanifolds may no longer be a submanifold. Also $N^{\#}\left(f_{1}, f_{2}\right)=0$ if $\omega^{\#}\left(f_{1}, f_{2}\right)=0$, but the converse may possibly not hold in generalnulbordisms of individual coincidence components may intersect in $M \times I$.

However, in the stable range $m \leq 2 n-2, \omega^{\#}\left(f_{1}, f_{2}\right)$ contains precisely as much information as $\widetilde{\omega}\left(f_{1}, f_{2}\right)$ does, and $N^{\#}\left(f_{1}, f_{2}\right)=N\left(f_{1}, f_{2}\right)$.

Let us summarize, we have the (successively weaker) invariants $\omega^{\#}\left(f_{1}, f_{2}\right), \widetilde{\omega}\left(f_{1}, f_{2}\right)$, $\omega\left(f_{1}, f_{2}\right)$ and $\mu\left(\omega\left(f_{1}, f_{2}\right)\right)=$ Poincaré dual of the cohomological primary obstruction to deforming $f_{1}$ and $f_{2}$ away from one another (cf. $[8,3.3]$ ); they are related by the natural forgetful maps

$$
\Omega^{\#}\left(f_{1}, f_{2}\right) \stackrel{\text { stabilize }}{\longrightarrow} \Omega_{m-n}\left(E\left(f_{1}, f_{2}\right) ; \tilde{\varphi}\right) \stackrel{p_{*}}{\longrightarrow} \Omega_{m-n}(M ; \varphi) \stackrel{\mu}{\longrightarrow} H_{m-n}\left(M ; \tilde{\mathbb{Z}}_{\varphi}\right)
$$

(cf. Remark 4.2, (4.3), and (3.1)). Only $\omega^{\#}\left(f_{1}, f_{2}\right)$ and $\widetilde{\omega}\left(f_{1}, f_{2}\right)$ involve the path space $E\left(f_{1}, f_{2}\right)$, thus allowing the definition of the Nielsen numbers $N^{\#}\left(f_{1}, f_{2}\right)$ and $N\left(f_{1}, f_{2}\right)$.

Example 4.3 the classical dimension setting $m=n$. Here the coincidence set

$$
C\left(f_{1}, f_{2}\right)=\coprod_{A \in \pi_{0}\left(E\left(f_{1}, f_{2}\right)\right)} \tilde{g}^{-1}(A)
$$

consists generically of isolated points (in this very special situation the stabilizing map and the Hurewicz homomorphism $\mu$ in (4.10) lead to no significant loss of information).

In our approach, each Nielsen class is expressed as an inverse image of some path component $A$ of $E\left(f_{1}, f_{2}\right)$ (compare Proposition 2.1). The corresponding index

$$
\tilde{\omega}_{A}\left(f_{1}, f_{2}\right) \in \Omega_{0}(A ; \tilde{\varphi} \mid A) \cong \begin{cases}\mathbb{Z} & \text { if } \omega_{1}(\tilde{\varphi} \mid A)=0, \\ \mathbb{Z}_{2} & \text { else, }\end{cases}
$$


(cf. Lemma 3.2) lies in $\mathbb{Z}_{2}$ precisely if $w_{1}(\tilde{\varphi} \mid A) \neq 0$ or, equivalently, if for some (and hence all) $x_{0} \in \tilde{g}^{-1}(A)$ there exists a class $\alpha \in \pi_{1}\left(M, x_{0}\right)$ such that $f_{1 *}(\alpha)=f_{2 *}(\alpha)$ but $w_{1}(M)(\alpha) \neq f_{1}^{*}\left(w_{1}(N)\right)(\alpha)$ (cf. [12, 5.2]; this agrees with the criterion quoted in [1, page 53 lines 5-6]). If $\pi_{1}(N)$ is commutative, then either the indices of all Nielsen classes are integers, or they all lie in $\mathbb{Z}_{2}$. However, it is easy to construct examples (e.g., involving maps from the Klein bottle to the punctured torus) where both types of path components $A \in \pi_{0}\left(E\left(f_{1}, f_{2}\right)\right)$ occur.

In any case our approach makes it clear from the outset where the indices of Nielsen classes must take their values.

In the setting of fixed point theory (where $f_{2}$ is the identity map on $M=N$ ) the transition from the $\tilde{\omega}$ - to the $\omega$-invariant (cf. (4.6) and (4.7)) which forgets the path space $E\left(f_{1}, f_{2}\right)$ parallels the transition from Nielsen to Lefschetz theory-with all the loss of information which this entails.

\section{Finiteness conditions for the minimum number $\operatorname{MC}\left(f_{1}, f_{2}\right)$}

Consider the following possible conditions concerning the invariants defined in (1.2), (4.6), and (4.7):

(C1) $\mathrm{MC}\left(f_{1}, f_{2}\right) \leq 1$;

(C2) $\mathrm{MC}\left(f_{1}, f_{2}\right)$ is finite ;

(C3) $\widetilde{\omega}\left(f_{1}, f_{2}\right)$ lies in the image of the homomorphism

$$
i_{E *}:=\bigoplus_{A} i_{A *}: \bigoplus_{A} \Omega_{m-n}^{\mathrm{fr}} \longrightarrow \bigoplus_{A} \Omega_{m-n}(A ; \tilde{\varphi} \mid)=\Omega_{m-n}\left(E\left(f_{1}, f_{2}\right) ; \tilde{\varphi}\right),
$$

where direct summation is taken over all $A \in \pi_{0}\left(E\left(f_{1}, f_{2}\right)\right)$ and $i_{A *}$ is induced by the inclusion of a point $z_{A}$ into the path component $A$ (and by a local orientation of $\tilde{\varphi}$ at $\left.z_{A}\right)$;

(C4) $\omega\left(f_{1}, f_{2}\right)$ lies in the image of a similarly defined homomorphism

$$
i_{*}: \Omega_{m-n}^{\mathrm{fr}} \longrightarrow \Omega_{m-n}(M ; \varphi) .
$$

Proposition 5.1. Each of the first three conditions implies the next one.

Proof. Assume that the coincidence set $C\left(f_{1}, f_{2}\right)$ is finite. If a generic pair $\left(f_{1}^{\prime}, f_{2}^{\prime}\right)$ approximates $\left(f_{1}, f_{2}\right)$ closely enough then each component of $C\left(f_{1}^{\prime}, f_{2}^{\prime}\right)$ lies in a ball neighbourhood of some $x \in C\left(f_{1}, f_{2}\right)$; moreover the corresponding paths which occur in the construction of $\widetilde{\omega}\left(f_{1}, f_{2}\right)$ lie entirely in a ball neighbourhood of $y=f_{1}(x)=f_{2}(x)$ and hence can be contracted into the constant path at $y$. Thus (C2) implies (C3). The proposition follows.

Our coincidence invariants $\widetilde{\omega}\left(f_{1}, f_{2}\right)$ and $\omega\left(f_{1}, f_{2}\right)$ project to the obstructions

$$
\begin{aligned}
& {\left[\tilde{\omega}\left(f_{1}, f_{2}\right)\right] \in \operatorname{coker}\left(i_{E *}\right),} \\
& {\left[\omega\left(f_{1}, f_{2}\right)\right] \in \operatorname{coker} i_{*},}
\end{aligned}
$$

which must vanish if $\operatorname{MC}\left(f_{1}, f_{2}\right)$ is to be finite. 
For $m-n=0$ these cokernels are trivial, $\operatorname{MC}\left(f_{1}, f_{2}\right)$ is actually finite and each integer $d \geq 0$ can occur as the value of this minimum number for a suitable pair of maps (e.g., for self maps of degrees $d$ and 0 on $S^{1}$ ).

If $m-n=1$ the cokernels in (5.3) are isomorphic_-via the Hurewicz homomorphism $\mu$ (cf. (3.1)) 一 to $H_{1}\left(E\left(f_{1}, f_{2}\right) ; \tilde{\mathbb{Z}}_{\tilde{\varphi}}\right)$ and $H_{1}\left(M ; \tilde{\mathbb{Z}}_{\varphi}\right)$, respectively, (compare Lemma 3.2). In fact $\mu$ vanishes on the image of $i_{E *}$ and of $i_{*}$, respectively, whenever $m-n \geq 1$, but in general the resulting homomorphisms on the cokernels will not be injective when $m-n>$ 1 (cf. $[12,9.3])$.

Remark 5.2. The finiteness criterion in Proposition 5.1 can be sharpened to yield a nonstabilized version involving $\omega^{\#}\left(f_{1}, f_{2}\right)$.

For self-coincidences there is a partial converse of Proposition 5.1.

Theorem 5.3. If $m<2 n-2$ and $f_{1}$ is homotopic to $f_{2}$, then the four conditions (C1)-(C4) are equivalent.

Proof. These conditions are compatible with homotopies of $f_{1}$ and $f_{2}$ (cf. [12, 3.3] and the discussion following (4.4)). Hence we may assume that $f_{1}=f_{2}=: f$.

Recall that the self-coincidence invariant $\omega(f, f)$ is just the singularity obstruction $\omega\left(\stackrel{\mathbb{R}}{\sim}, f^{*}(T N)\right)$ to sectioning the vector bundle $f^{*}(T N)$ over $M$ without zeroes (cf. [11, Theorem 2.2]).

Now assume that $m<2 n-2$ and $\omega(f, f)=i_{*}\left(\omega_{0}\right)$ for some $\omega_{0} \in \Omega_{m-n}^{\mathrm{fr}} \cong \pi_{m-n}^{S}$. Then there exists a map $u^{\partial}: S^{m-1} \rightarrow S^{n-1}$ whose (stable) Freudenthal suspension corresponds to $\omega_{0}$. Now consider the trivial bundle $f^{*}(T N) \mid B^{m}$ over some compact ball $B^{m}$ in $M$ and interpret $u^{\partial}$ as a nowhere zero section over $\partial B^{m}=S^{m-1}$.

We will extend $u^{\partial}$ to a section $u$ of $f^{*}(T N)$ over all of $M$ which vanishes only in the centre point of $B^{m}$. Over the ball $B^{m}$ we use the obvious "concentric" extension. Note, however, that generically the zero set of any extension of $u^{\partial}$ over $B^{m}$ is a framed manifold which represents $\omega_{0}$. Thus a generic extension of $u^{\partial}$ to the complement $M-B^{m}$ must have a nulbordant manifold of zeroes (representing $\omega(f, f)-i_{*}\left(\omega_{0}\right)=0$ ). According to [9, Theorem 3.7] these zeroes can be removed altogether.

The resulting section $u$ of $f^{*}(T N)$ allows us to construct a "small" homotopy of $f$ : for every $x \in M$ just deform $f(x)$ somewhat in the direction of the tangent vector $u(x) \in$ $T_{f(x)}(N)$. We obtain a map which has only one coincidence point with $f$.

\section{The examples of the introduction}

In view of the self-coincidence theorem in [11] and of our Theorem 5.3 the first example is a special case of the following proposition.

Proposition 6.1. Let $\xi$ be an oriented real plane bundle over a closed smooth connected manifold $N$ and let $f: M:=S(\xi) \rightarrow N$ denote the projection of the corresponding unit circle bundle. Then, the following exist:

(i) the self-coincidence invariant $\omega(f, f)$ (cf. (4.7)) vanishes if and only if the Euler number $\chi(N)$ of $N$ lies in $e(\xi)\left(H_{2}(N ; \mathbb{Z})\right) \subset \mathbb{Z}$ and $\chi(N)$ is even; here $e(\xi)$ denotes the Euler class of $\xi$, 
(ii) the finiteness obstruction $\mu(\omega(f, f)) \simeq[\omega(f, f)]$ (cf. (5.3) and the subsequent discussion in Section 5) vanishes if and only if $\chi(N) \in e(\xi)\left(H_{2}(N ; \mathbb{Z})\right)$.

Proof. We will extend the arguments of [11, Section 4]. Consider the commuting diagram

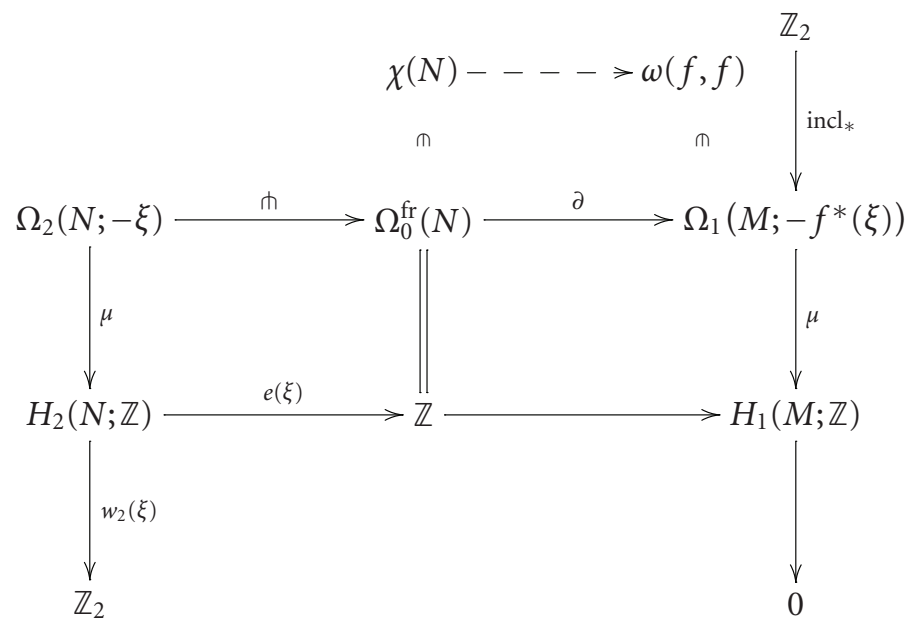

Here the vertical exact sequences are as described in Lemma 3.2. The horizontal lines are exact Gysin sequences of $\xi$ in normal bordism and in homology (or, equivalently, oriented bordism), compare [9, 9.20 and 9.4]. As was shown in [11, Section 4], we have $\omega(f, f)=\chi(N) \cdot \partial(1)$. Since the Stiefel-Whitney class $w_{2}(\xi)$ is the $\bmod 2 \operatorname{reduction}$ of $e(\xi)$, the proposition follows.

Next let us examine Example 1.5. If $r \geq 2 k \geq 2$ then according to the theorem in the introduction of [11] only the "highest order part" 1.11 of the complete obstruction $\omega(f, f)$ (to deforming $f$ away from itself) survives. Thus the finiteness obstruction $[\omega(f, f)]$ (cf. (5.3)) vanishes. If also $k \geq 2$ then it follows from Theorem 5.3 that $\mathrm{MC}(f, f)$ (and hence also $\operatorname{MCC}(f, f))$ equals 0 or 1 according to whether $\omega(f, f)$ vanishes or not. It requires using deep results of homotopy theory and of other branches of algebraic topology to decide which of the two values occur actually, but it can be done at least for $k \leq 10$ (cf. [11, Section 3]). However, the case $k=1$ (where we deal with the standard projection from $S^{r-1}$ to real projective space) is elementary.

Next we turn to Example 1.6 where $N=\left(S^{1}\right)^{n}$. Use the Lie group structure to replace $\left(f_{1}, f_{2}\right)$ by the pair $(f, *)$ which consists of the quotient $f=f_{1} \cdot f_{2}^{-1}$ and of the constant map taking values at the unit element of $\left(S^{1}\right)^{n}$. This does not change the coincidence sets and data significantly.

Since each torus $\left(S^{1}\right)^{k}$ is a $K\left(\mathbb{Z}^{k}, 1\right)$-space the homotopy class of $f$ is determined by $f_{*}$ : $H_{1}(M ; \mathbb{Z}) \rightarrow H_{1}\left(\left(S^{1}\right)^{n} ; \mathbb{Z}\right)$. Moreover, if the image of $f_{*}$ has rank $k<n$, then $f$ factors up to homotopy through the lower-dimensional torus $\left(S^{1}\right)^{k}$ and hence through $\left(S^{1}\right)^{n}-\{*\}$. On the other hand, if the image of $f_{*}$ has rank $n$ (or, equivalently, the Reidemeister set $R(f, *) \cong \operatorname{coker} f_{*}$ is finite) then-according to Remark 1.3-all Reidemeister classes are essential and hence $N(f, *)=\# R(f, *)$, provided $\widetilde{\omega}(f, *) \neq 0$. This holds, in particular, 
if the invariant

$$
\omega(\text { coll } \circ f, *) \in \Omega_{m-n}(M ;-T M)
$$

which corresponds to the bordism class of the stably coframed manifold $C(f, *)=$ $f^{-1}(\{*\}) \subset M$, is nontrivial. Here the map

$$
\text { coll }: N=\left(S^{1}\right)^{n} \longrightarrow N /(N-\stackrel{\circ}{B}) \cong S^{n}
$$

collapses the complement of an open ball to a point. The induced cohomology homomorphisms coll ${ }^{*}$, and $(f \circ \text { coll })^{*}$, respectively, map a generator of $H^{n}\left(S^{n} ; \mathbb{Z}\right)$ to the cup product $\iota_{1} \cdots \iota_{n} \in H^{n}\left(\left(S^{1}\right)^{n} ; \mathbb{Z}\right)$, and to the Poincaré dual of $\mu(\omega(f, *))$, respectively, (compare (4.10) and $[8,3.3])$. Our claims concerning Example 1.6 in the introduction follow now from Section 5.

Finally note that the facts described in Example 1.7 follow mainly from the discussion in [12] (see 1.14-1.17 as well as Sections 7 and 8); the calculation of $\Omega_{1}^{\mathrm{fr}}\left(\Omega S^{2}\right)$ can be understood easily with the help of our Lemma 3.2.

Let us put the role of the path space $E\left(f_{1}, f_{2}\right)$ and its influence on the relative strength of our invariants into perspective (compare diagram (4.10)).

In the self coincidence situation $f_{1}=f_{2}:=f$ the fibration $p: E(f, f) \rightarrow M$ allows a global section $s$ by constant paths; therefore $\tilde{\omega}(f, f)=s_{*}(\omega(f, f))$ is precisely as strong as the (usually much weaker) invariant $\omega(f, f)$ which does not involve any path space data. As Examples 1.4 and 1.5 illustrate, $\omega(f, f)$ may nevertheless capture decisive and very delicate information (which is also registered to some extend by the Nielsen number $N(f, f)$ in spite of the fact that it can take only the values 0 and 1$)$.

In Example 1.6 our path space approach serves to decompose coincidence sets into Nielsen classes. However, it does not seem to enrich the higher-dimensional homotopy theoretical aspects of their data very much (as the torus is aspherical; compare Proposition 2.1). Still, all natural numbers can occur here as Nielsen numbers of suitable maps $f_{1}$ and $f_{2}$.

In contrast, in Example 1.7 the higher-dimensional topology of $E\left(f_{1}, f_{2}\right)$ turns out to be potentially very rich (e.g., when $N=S^{n}, n \geq 2$ ) and able to capture much more than just the decomposition into Nielsen classes.

\section{Acknowledgment}

This work was supported in part by the Deutsche Forschungsgemeinschaft and AARMS (Canada).

\section{References}

[1] S. A. Bogaty̆, D. L. Gonçalves, and H. Zieschang, Coincidence theory: the minimization problem, Proceedings of the Steklov Institute of Mathematics 225 (1999), no. 2, 45-77.

[2] R. B. S. Brooks, On removing coincidences of two maps when only one, rather than both, of them may be deformed by a homotopy, Pacific Journal of Mathematics 40 (1972), no. 1, 45-52. 
[3] R. F. Brown, Wecken properties for manifolds, Nielsen Theory and Dynamical Systems (Mass, 1992), Contemp. Math., vol. 152, American Mathematical Society, Rhode Island, 1993, pp. 921.

[4] P. E. Conner and E. E. Floyd, Differentiable Periodic Maps, Ergebnisse der Mathematik und ihrer Grenzgebiete, N. F., vol. 33, Academic Press, New York; Springer, Berlin, 1964.

[5] O. Cornea, G. Lupton, J. Oprea, and D. Tanré, Lusternik-Schnirelmann Category, Mathematical Surveys and Monographs, vol. 103, American Mathematical Society, Rhode Island, 2003.

[6] J.-P. Dax, Étude homotopique des espaces de plongements, Annales Scientifiques de l'École Normale Supérieure. Quatrième Série (4) 5 (1972), 303-377 (French).

[7] A. Dold and D. L. Gonçalves, Self-coincidence of fibre maps, Osaka Journal of Mathematics 42 (2005), no. 2, 291-307.

[8] D. L. Gonçalves, J. Jezierski, and P. Wong, Obstruction theory and coincidences in positive codimension, Bates College, preprint, 2002.

[9] U. Koschorke, Vector Fields and Other Vector Bundle Morphisms—a Singularity Approach, Lecture Notes in Mathematics, vol. 847, Springer, Berlin, 1981.

[10] L Linking and coincidence invariants, Fundamenta Mathematicae 184 (2004), 187-203.

[11] Selfcoincidences in higher codimensions, Journal für die Reine und Angewandte Mathematik 576 (2004), 1-10.

[12] _ Nielsen coincidence theory in arbitrary codimensions, to appear in to appear in Journal f́ur die Reine und Angewandte Mathematik, 2003, http:/www.math.uni-siegen.de/topology/ publications.html.

[13] _ Nonstabilized Nielsen coincidence invariants and Hopf-Ganea homomorphisms, preprint, 2005, http://www.math.uni-siegen.de/topology/publications.html.

[14] H. Toda, Composition Methods in Homotopy Groups of Spheres, Annals of Mathematics Studies, no. 49, Princeton University Press, New Jersey, 1962.

Ulrich Koschorke: Universität Siegen, Emmy Noether Campus, Walter-Flex Street 3, D-57068 Siegen, Germany

E-mail address: koschorke@mathematik.uni-siegen.de 